\title{
An overview of sugarcane brown rust in Cuba
}

María La 01, María Francisca Perera ${ }^{2}$, Romina Priscila Bertani2 , Ricardo Acevedo ${ }^{1}$, Marta Eugenia Arias ${ }^{3,4}$, Mario Alberto Casas ${ }^{1}$, Juana Pérez ${ }^{1}$, Yaquelín Puchades ${ }^{1}$, Eida Rodríguez ${ }^{1}$, Isabel Alfonso ${ }^{1}$, Atilio Pedro Castagnaro²*

\begin{abstract}
${ }^{1}$ Sugarcane Research Institute, carretera CUJAE km 11/2Boyeros, La Havana - Cuba.

${ }^{2}$ Agroindustrial Technology Institute of Northwest Argentine' Agroindustrial Experimental Station "Obispo Colombres" National Council of Scientific and Technical Research, Av. William Cross, 3150 - Las Talitas, Tucumán - Argentina. ${ }^{3}$ National University of Tucuman/Faculty of Natural Sciences/ Miguel Lillo Institute, Miguel Lillo, 205 - San Miguel de Tucumán, Tucumán - Argentina.

${ }^{4}$ National University of Catamarca/Faculty of Natural

Sciences, Av. Belgrano, 300 - San Fernando del Valle de

Catamarca, Catamarca - Argentina.

*Corresponding author <atilio@eeaoc.org.ar>
\end{abstract}

Edited by: Claudio Marcelo Gonçalves de Oliveira

Received September 20, 2016

Accepted February 20, 2017
ABSTRACT: Multiple pathogens affect sugarcane, among them Puccinia melanocephala, the causal agent of brown rust. This disease was first reported in Cuba in 1979 when it was responsible for a severe attack on the main sugarcane variety B4362. The aims of the present study were to give an overview of sugarcane brown rust in Cuba and show the current disease situation in the country. A retrospective analysis regard to sugarcane cultivar composition resistant to brown rust in Cuba was carried out. In addition 154 genotypes, including the most used progenitors in the breeding program and commercial varieties were evaluated under natural infection conditions. The identity of $P$. melanocephala was verified by Polymerase Chain Reaction (PCR) and by sequencing the ITS1 region. After the introduction of $P$. melanocephala into Cuba, the susceptible variety, B4362, was replaced by Ja60-5 which remained resistant until 1998 . Since 2002, a varietal policy supported by a governmental resolution establishing that any single cultivar cannot occupy more than $20 \%$ of the production area for each production company, local area and province, has been applied. Out of the genotypes evaluated, 49 showed resistance to the disease and 35 intermediate behavior, while 39 were susceptible and 31 , highly susceptible. $P$. melanocephala was detected by PCR in all symptomatic samples and its identify confirmed by sequencing the ITS1 region. The adopted measurement together with permanent phytosanitary monitoring and commercial release of resistant or intermediate cultivars succeeded in avoiding any new epidemic. Inoculum pressure was reduced, even on susceptible and highly susceptible varieties since, by resolution, they cannot occupy more than $10 \%$ of the planted area.

Keywords: Puccinia melanocephala, varietal mosaic, resistance

\section{Introduction}

Multiple pathogens affect sugarcane, among them Puccinia melanocephala H. \& P. Sydow, causal agent of brown rust. This disease, which has spread worldwide in sugarcane growing areas (Glynn et al., 2010), was reported in the American continent in 1978, in the Dominican Republic (Purdy et al., 1985). The following year it was detected in Cuba where it was responsible for a severe attack on the B4362 variety which occupied around $40 \%$ of the sugarcane area of the country at that time.

$P$. melanocephala causes reduced plant growth as expressed by the reduction of the length and number of stalks per plant (Hoy and Hollier, 2009). Thus, susceptible cultivars register losses from 10 to $40 \%$ around the world (Costet et al., 2012). This pathogen also generates considerable economic impact in breeding programs since resistance to brown rust is one of the selection criteria that leads to the elimination of precommercial susceptible clones. However, an adequate management of the varietal composition could reduce the infected areas (Raid and Comstock, 2000).

Rust typically produces cyclic epidemic explosions, which are dependent on host susceptibility, favorable environmental conditions and the inocula. The permanence of the pathogen in the field makes possible the emergence of more virulent strains, which could cause resistance breakdown in certain sugarcane genotypes. The introduction of $P$. melanocephala to Cuba al- lowed the highly susceptible B4362 to become severely infected. This variety was rapidly replaced by Ja60-5 which remained resistant to $P$. melanocephala until 1998. Since then, several varieties have had their resistance broken by $P$. melanocephala and have needed to be replaced.

The aims of the present work were to give an overview of sugarcane brown rust in Cuba and show the current disease situation in the country.

\section{Materials and Methods}

\section{Retrospective analysis of the disease in production areas}

Information on the behavior of sugarcane cultivars against $P$. melanocephala and the modification of percentages of planted area with susceptible cultivars from 1970 to the present were compiled from historical records of the sugarcane breeding program of the Sugarcane Research Institute of Cuba (INICA).

In addition, a disease severity index for each year from 2000 was calculated according to the equation proposed by Townsend and Heuberger (1943) as follows: $D S=\left(\sum\left(a^{*} b\right) / n * k\right) * 100$, where: $a$ : number of plants within each infection category on a scale rating from 1 (highly resistant) to 5 (highly susceptible) as proposed by Rao et al. (1996) with minor modifications; $b$ : degree of infection according to the scale; $n$ : total number of plants screened and $k$ : highest value on the scale $(5$, in this case). 
Evaluation of resistance of the progenitors frequently used in the breeding program and major commercial cultivars

Field testing for brown rust susceptibility was carried out in an experimental field in the municipality of Jovellanos, in Matanzas province in Cuba $\left(22^{\circ} 47^{\prime} 53^{\prime \prime} \mathrm{N}\right.$, $81^{\circ} 08^{\prime} 33^{\prime \prime}$ W, 25.23 m.a.s.l.) under high inoculum pressure. One hundred and fifty four progenitors used in the breeding program that included 13 major commercial cultivars were planted in a randomized block design using three replications, each one consisting of individual 6-meter-long row plots. Cultivar B4362, which is very susceptible to brown rust, was planted as borders surrounding the trial, as well as repeatedly inside the experimental area in order to maximize infection. Beginning at 45 days after germination, $3+$ leaves of 10 plants per cultivar were randomly collected from the center of the plot for evaluating rust severity until the plants were 6 months old. Maximum ratings were considered for each genotype.

Brown rust reaction was scored by using a scale proposed by Rao et al. (1996) with minor modifications with ratings varying from 1 (highly resistant) to 5 (highly susceptible) based on visual assessment of disease symptoms (foliar area occupied by pustules). Standard cultivars representing each category on the scale were evaluated (B4362 - Highly Susceptible, HS: > 30 \%; My5514 and Ja60-5 - Susceptible, S: > $15 \leq 30 \%$; C334-64 - Intermediate, INT: $>5 \leq 15 \%$; Ja64-11 - Resistant, R: $>0 \leq$ $5 \%$; and PR980 - Highly Resistant, HR: 0 \%). A regression equation between disease symptoms and ratings was obtained and it was used to determine the behavior (rating) of the tested genotypes.

\section{Detection and identification of the causal agent of sugarcane brown rust}

In order to verify the rust pathogen's identity in Cuba, 94 symptomatic sugarcane samples were collected from 14 localities in three different years (Table 1, Figure 1). Total nucleic acids were extracted by using the Cetyl trimethylammonium bromide (CTAB) technique (Aljanabi et al., 1999), with minor modifications. An additional cleaning step with phenol was included to obtain a better quality of nucleic acid. Samples were amplified by PCR with the primer pair $\mathrm{PkPmF} / \mathrm{PkPmR}$ that amplifies the ITS1 region and allows for differentiating between $P$. melanocephala and $P$. kuehnii according to the size of the fragment obtained, 585 and $606 \mathrm{bp}$, respectively (Glynn et al., 2010).

Three samples were randomly selected including sugarcane genotypes with different behavior to brown rust B4362.MT.10, My5514.MT.10, and PR980.MT.10: HS, S and HR to brown rust, respectively. In the case of PR980 only chlorotic lesions could be found. Samples were amplified with the pair of primers $\mathrm{PmF} / \mathrm{PmR}$, specific to P. melanocephala (Glynn et al., 2010); the amplified fragments were purified and sequenced in an automatic DNA sequencer Abi (3130xl Genetic Analyzer, Hitachi). The results obtained from the sequencing in
Table 1 - Sugarcane samples collected for Puccinia melanocephala molecular detection.

\begin{tabular}{|c|c|c|c|}
\hline $\begin{array}{l}\text { Year of } \\
\text { sampling }\end{array}$ & Province & Genotype & Code \\
\hline \multirow{32}{*}{2010} & \multirow{6}{*}{ Camagüey } & B4362 & B4362.CM.10 \\
\hline & & C333-64 & C333-64.CM.10 \\
\hline & & Ja64-19 & Ja64-19.CM.10 \\
\hline & & My5514 & My5514.CM.10 \\
\hline & & PR980 & PR980.CM.10 \\
\hline & & SP70-1284 & SP70-1284.CM.10 \\
\hline & \multirow{6}{*}{ Habana } & B4362 & B4362.H.10 \\
\hline & & C333-64 & C333-64.H.10 \\
\hline & & Ja64-19 & Ja64-19.H.10 \\
\hline & & My5514 & My5514.H.10 \\
\hline & & PR980 & PR980.H.10 \\
\hline & & SP70-1284 & SP70-1284.H.10 \\
\hline & \multirow{7}{*}{ Matanzas } & B4362 & B4362.MT.10 \\
\hline & & C333-64 & C333-64.MT.10 \\
\hline & & \multirow{2}{*}{ Ja64-19 } & Ja64-19.MT.10(1) \\
\hline & & & Ja64-19.MT.10(2) \\
\hline & & My5514 & My5514.MT.10 \\
\hline & & PR980 & PR980.MT.10 \\
\hline & & SP70-1284 & SP70-1284.MT.10 \\
\hline & \multirow{7}{*}{ Santiago de Cuba } & \multirow{2}{*}{ B4362 } & B4362.ST.10(1) \\
\hline & & & B4362.ST.10(2) \\
\hline & & C333-64 & C333-64.ST.10 \\
\hline & & Ja64-19 & Ja64-19.ST.10 \\
\hline & & My5514 & My5514.ST.10 \\
\hline & & PR980 & PR980.ST.10 \\
\hline & & SP70-1284 & SP70-1284.ST.10 \\
\hline & \multirow{6}{*}{ Villa Clara } & B4362 & B4362.VC.10 \\
\hline & & C333-64 & C333-64.VC.10 \\
\hline & & Ja64-19 & Ja64-19.VC.10 \\
\hline & & My5514 & My5514.VC.10 \\
\hline & & PR980 & PR980.VC.10 \\
\hline & & SP70-1284 & SP70-284.VC.10 \\
\hline \multirow{18}{*}{2012} & \multirow{4}{*}{ Ciego de Ávila } & \multirow{18}{*}{ B4362 } & B4362.CA.12(1) \\
\hline & & & B4362.CA.12(2) \\
\hline & & & B4362.CA.12(3) \\
\hline & & & B4362.CA.12(4) \\
\hline & \multirow{4}{*}{ Cienfuegos } & & B4362.CF.12(1) \\
\hline & & & B4362.CF.12(2) \\
\hline & & & B4362.CF.12(3) \\
\hline & & & B4362.CF.12(4) \\
\hline & \multirow{6}{*}{ Camagüey } & & B4362.CM.12(1) \\
\hline & & & B4362.CM.12(2) \\
\hline & & & B4362.CM.12(3) \\
\hline & & & B4362.CM.12(4) \\
\hline & & & B4362.CM.12(5) \\
\hline & & & B4362.CM.12(6) \\
\hline & \multirow{4}{*}{ Granma } & & B4362.GR.12(1) \\
\hline & & & B4362.GR.12(2) \\
\hline & & & B4362.GR.12(3) \\
\hline & & & B4362.GR.12(4) \\
\hline
\end{tabular}

Continue... 
Table 1 - Continuation.

\begin{tabular}{|c|c|c|c|}
\hline & \multirow{2}{*}{ Guantánamo } & & B4362.GT.12(1) \\
\hline & & & B4362.GT.12(2) \\
\hline \multirow{20}{*}{2012} & \multirow{3}{*}{ Holguín } & \multirow{20}{*}{ B4362 } & B4362.HL.12(1) \\
\hline & & & B4362.HL.12(2) \\
\hline & & & B4362.HL.12(3) \\
\hline & Las Tunas & & B4362.LT.12 \\
\hline & \multirow{3}{*}{ Matanzas } & & B4362.MT.12(1) \\
\hline & & & B4362.MT.12(2) \\
\hline & & & B4362.MT.12(3) \\
\hline & Mayabeque & & B4362.MY.12 \\
\hline & \multirow{4}{*}{ Pinar del Río } & & B4362.PR.12(1) \\
\hline & & & B4362.PR.12(2) \\
\hline & & & B4362.PR.12(3) \\
\hline & & & B4362.PR.12(4) \\
\hline & Sancti Spíritus & & B4362.SS.12 \\
\hline & \multirow{4}{*}{ Santiago de Cuba } & & B4362.ST.12(1) \\
\hline & & & B4362.ST.12(2) \\
\hline & & & B4362.ST.12(3) \\
\hline & & & B4362.ST.12(4) \\
\hline & \multirow{3}{*}{ Villa Clara } & & B4362.VC.12(1) \\
\hline & & & B4362.VC.12(2) \\
\hline & & & B4362.VC.12(3) \\
\hline \multirow{22}{*}{2013} & \multirow{3}{*}{ Ciego de Ávila } & B4362 & B4362.CA.13 \\
\hline & & C120-78 & C120-78.CA.13 \\
\hline & & My5514 & My5514.CA.13 \\
\hline & Matanzas & B4362 & B4362.MT.13 \\
\hline & \multirow{10}{*}{ Mayabeque } & \multirow{2}{*}{ B4362 } & B4362.MY.13(1) \\
\hline & & & B4362.MY.13(2) \\
\hline & & C120-78 & C120-78.MY.13 \\
\hline & & C333-64 & C333-64.MY.13 \\
\hline & & \multirow{2}{*}{ C86-456 } & C86-456.MY.13(1) \\
\hline & & & C86-456.MY.13(2) \\
\hline & & Ja60-5 & Ja60-5.MY.13 \\
\hline & & \multirow{2}{*}{ My5514 } & My5514.MY.13(1) \\
\hline & & & My5514.MY.13(2) \\
\hline & & PR980 & PR980.MY.13 \\
\hline & Santiago de Cuba & B4362 & B4362.ST.13 \\
\hline & \multirow{7}{*}{ Villa Clara } & \multirow{2}{*}{ B4362 } & B4362.VC.13(1) \\
\hline & & & B4362.VC.13(2) \\
\hline & & Ja60-5 & Ja60-5.VC.13 \\
\hline & & \multirow{2}{*}{ My5514 } & My5514.VC.13(1) \\
\hline & & & My5514.VC.13(2) \\
\hline & & \multirow{2}{*}{ PR980 } & PR980.VC.13(1) \\
\hline & & & PR980.VC.13(2) \\
\hline
\end{tabular}

both directions were used to generate the consensus sequence by using the DNAman software program. Sequences were aligned by the ClustalX program (Thompson et al., 1997) and their phylogeny determined by the MEGA6 software program (Koichiro et al., 2013) using the neighbor-joining option with a bootstrap analysis of 1,000 random replications. Certain sequences deposited in GenBank belong to isolates of $P$. melanocephala from other countries and several species of the Puccinia genus were included in the analysis.

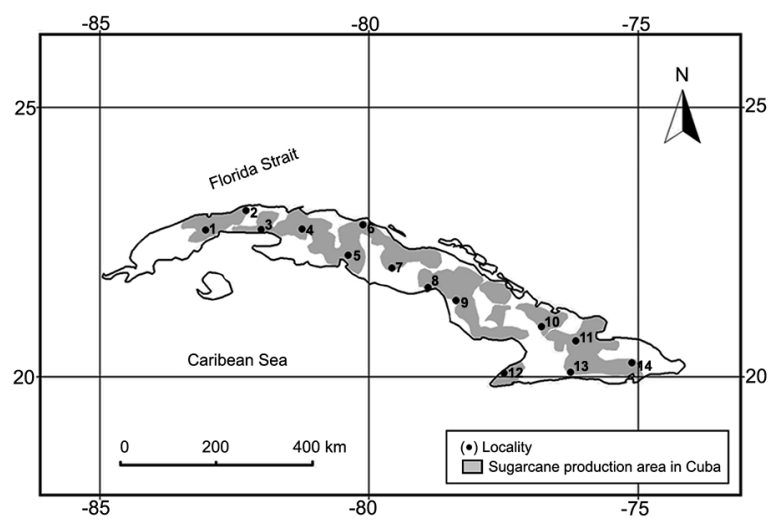

Figure 1 - Location of field trials monitored for brown rust in sugarcane during the 2010, 2012 and 2013 seasons. 1: Pinar del Río (22.73-83.03), 2: Habana (23.09-82.28), 3: Mayabeque (22.74-82), 4: Matanzas (22.75-81.24), 5: Cienfuegos (22.2680.39), 6: Villa Clara (22.83-80.11), 7: Sancti Spirítus (22.0279.57), 8: Ciego de Ávila (21.67-78.91), 9: Camagüey (21.4278.38), 10: Las Tunas (20.94-76.79), 11: Holguin (20.68-76.16), Granma (20.06-77.49), 13: Santiago de Cuba (20.09-76.25) and 14: Guantánamo (20.26-75-11), in Cuba.

\section{Results}

\section{Retrospective analysis of the disease in production} areas

In 1970, cultivar B4362, which was subsequently determined to be highly susceptible to brown rust after its introduction in 1979, occupied between 45 and $55 \%$ of Cuba's growing area. In 1979, B4362 was replaced by Ja60-5 that was resistant to brown rust at that time. The area planted with Ja60-5 increased rapidly, reaching 60 $\%$ of the total area until 1999 when it had its resistance broken.

Since 2002, a varietal policy supported by a governmental resolution (616/2014) of the Agriculture Ministry of Cuba, was applied to avoid the risk of new outbreaks of the disease. This policy established that no one cultivar can occupy more than $20 \%$ of the production area for each production company, local area and province. This measurement together with permanent phytosanitary monitoring of the cultivars and commercial release by the local sugarcane breeding program of resistant and intermediate genotypes has succeeded in avoiding new epiphytotics, reducing inoculum pressure on even susceptible and highly susceptible varieties which, according to the above mentioned governmental resolution (616/2014), cannot occupy more than $10 \%$ of the planted area. This is evidenced by the reduction in the disease severity index over the years, as presented in Figure 2.

As regards cultivars that have occupied significant production areas since 2000, C323-68 (resistant at that time) was the leader reaching $20 \%$ until 2005 when its resistance was broken down. Since then until present, 


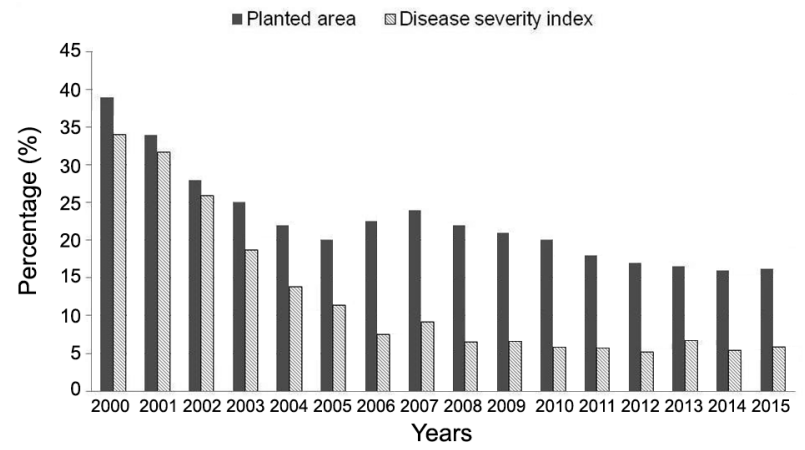

Figure 2 - Evolution of the sugarcane production area occupied by susceptible cultivars to brown rust and disease severity index from 2000 to 2015 in Cuba.

as a result of the measures adopted in Cuba, there is a varietal mosaic represented by 98 cultivars, as was demonstrated by the census carried out in June of 2015 by the Sugarcane Research Institute of Cuba (INICA). Out of these cultivars, 85 were obtained by the National Breeding Program and occupy $83 \%$ of the planted area, whereas the rest of the genotypes were introduced from different breeding centers in the world and occupy $17 \%$ of the Cuban sugarcane area.

Figure 2 showed the evolution of the commercial production area occupied by susceptible cultivars to brown rust in Cuba. In 2015, the cultivars covering the largest production area were: C86-56 (6 \%, R), C90-469 (5 \%, INT), C323-68 (5 \%, HS), C90-317 (4 \%, R) and C86-503 (3\%, S). The resistance to brown rust of certain genotypes was broken down in certain localities and their planting was limited. However, the use of moderate susceptible cultivars plays an important role, considering that low concentration of inoculum has limited rust severity.

\section{Evaluation of resistance of the progenitors most frequently used in the breeding program and major commercial cultivars}

Performance of the standard cultivars corresponded to their historical ratings. These data were used to calculate the following regression equation between the percentage of foliar area occupied by pustules in trials and the historical ratings of the standard genotypes: Percentage of foliar area occupied by pustules $=15.668^{*}$ rating - 23.6, where the correlation coefficient calculated from the regression was 0.84 . From the equation, ratings for the tested varieties were determined on a 1-5 scale.

One hundred and fifty four progenitors most frequently used in the Cuban breeding program were evaluated to determine their behavior against $P$. melanocephala. Out of these, 49 showed resistance to brown rust, while 35 were intermediate, 39 susceptible and 31 highly susceptible. No genotype showed highly resistant behavior as much as the control PR980 variety (Table 2).
The brown rust reactions of the most commonly grown cultivars that are also used as progenitors by the breeding program are shown in Table 3 . These 13 cultivars occupy $36 \%$ of the total area planted with sugarcane in Cuba. Among them, HS and S genotypes (16\% of the total area) correspond to varieties introduced before the year 2000, whereas INT and R varieties were incorporated from 2002 in line with a new varietal policy.

\section{Detection and identification of the causal agent of brown rust}

Puccinia melanocephala was detected in all collected symptomatic samples by PCR. Three amplified fragments corresponding to the ITS1 region were purified, sequenced and deposited in the GenBank with the following accession numbers: KU180235, KU180236 and KU180237. A phylogenetic analysis was carried out including other available sequences at GenBank of $P$. melanocephala and other species of the Puccinia genus (Figure 3). All $P$. melanocephala sequences were grouped together.

\section{Discussion}

Several pathogens affect sugarcane production and cause devastating economic losses; therefore, it is necessary to establish limiting percentages in the development of cultivars to prevent the outbreak of disease. Thus, the occurrence of rust epidemics is conditioned by the direct relationship between the areas planted with highly susceptible sugarcane cultivars and the presence of the pathogen in environmental conditions favorable to its development, which lead to a high inoculum concentration that guarantees auto-infection (Agrios, 2005).

Brown rust sugarcane symptoms in Cuba are caused by the fungal pathogen $P$. melanocephala since its presence was confirmed by specific PCR amplification of DNA in all samples collected. As expected, considering that the sequence of the rDNA does not reveal the genetic pathogen diversity as it represents only a small fragment of the entire genome (Braithwaite et al., 2009), the ITS sequences of three different samples did not show any polymorphism. However, they were useful in verifying the rust pathogen's identity.

In Cuba, two main factors have prevented the development of new epidemics of brown rust: first, the replacement of highly susceptible varieties that could serve as inoculum sources, such as B4362 and Ja60-5; and second, the limit of $20 \%$ on the area occupied by a single variety, which could limit pathogen specialization. Even more, susceptible cultivars do not show severe symptoms indicating that the variety diversity can be limiting the buildup of inoculum.

Genetic resistance is the most efficient measurement in the implementation of brown rust control and represents a decisive selection criterion in sugarcane breeding programs. Resistance to this disease is considered hereditary quantitatively (Chu et al., 1982), so progress in breeding programs for brown rust resistance 
Table 2 - Behavior of progenitors more frequently used in Cuban breeding program against Puccinia melanocephala.

Behavior against $P$. melanocephala

$\begin{array}{ll}\text { Highly Susceptible } & \text { B37161, B42231, B4362, B51410, C1051-73, C120-78, C1616-75, C187-68, C2366-88, C237-80, C266-70, C323-68, C334-64, }\end{array}$ C568-75, C87-51, C94-60, CG127-45, Co281, Co7
Ja64-19, Ja64-20, My54129, My5724, NCo310

B41227, B45181, B45211, B6368, B77218, B77330, B80394, B8066, C227-59, C229-84, C86-12, C86-156, C86-251, C86-503,

$\begin{array}{ll}\text { Susceptible } & \text { C86-535, C88-356, C88-380, C88-553, C89-372, C90-105, C90-316, C91-301, C92-325, C92-514, Co419, Co421, CP27-108, } \\ & \text { CP29-103, CP68-1154, CP70-1133, CP72-2086, CP72-356, CSG87-508, Ja60-5, ML3-18, My5514, My5823, NA63-90, Phill6607, }\end{array}$ Pomex72, SP70-1284

Intermediate

B60267, B77311, B78505, B80250, C137-81, C147-76, C334-64, C85-154, C85-403, C86-407, C86-456, C86-502, C86-531, C87-135, C88-383, C88-523, C89-136, C89-161, C89-176, C90-101, C90-469, C90-501, C90-530, C91-367, C91-522, C92-203, C92-249, C92-26, C94-504, C97-59, CP56-59, CP65-392, CP70-1527, Mex66-1235, Mex68-200, P0J2878

C1566-75, C258-88, C311-86, C85-102, C85-277, C85-293, C85-473, C85-507, C86-410, C86-534, C86-551, C86-56, C86-602,

Resistant C87-134, C87-253, C88-381, C88-556, C89-137, C89-147, C89-148, C89-250, C89-504, C89-507, C89-509, C90-159, C90-317, C90-647, C91-115, C91-356, C92-524, C93-540, C93-567, C97-373, C06806, CP49-50, CP61-83, CR63-124, CSG86-509, CSG86510, CSG87-502, CSG87-506, CSR100-87, CSR71-88, Ja64-11, L56-23, L60-25, L68-90, M165-38, Mex60-1459, Mex65-1424

Highly Resistant PR980

In bold letters standard cultivars representing each category at the rating scale for scoring brown rust reaction.

Table 3 - Percentage of area occupied by major sugarcane varieties in Cuba in 2015, their progenitors and their behaviors against Puccinia melanocephala.

\begin{tabular}{lcclclc}
\hline Cultivar & \% area occupied & RR & $\begin{array}{c}\text { Female } \\
\text { progenitor }\end{array}$ & RR & $\begin{array}{c}\text { Male } \\
\text { progenitor }\end{array}$ & RR \\
\hline C86-56 & 6 & R & NC0310 & S & C187-68 & HS \\
C90-469 & 5 & INT & C87-51 & HS & Ja60-5 & S \\
C323-68 & 5 & HS & B4362 & HS & C87-51 & HS \\
C90-317 & 4 & R & C187-68 & HS & B6368 & S \\
C86-503 & 3 & S & C568-75 & HS & Ja60-5 & S \\
C89-147 & 2 & R & C236-51 & S & B45181 & S \\
C90-530 & 2 & INT & My5514 & S & C0421 & S \\
C86-156 & 2 & S & C16-56 & S & C87-51 & HS \\
C87-51 & 2 & HS & Co281 & HS & P0J2878 & INT \\
C1051-73 & 2 & HS & B42231 & HS & C431-62 & INT \\
C89-176 & 1 & INT & NC0310 & S & C187-68 & HS \\
My5514 & 1 & S & CP34-79 & S & B45181 & S \\
C120-78 & 1 & HS & C0421 & S & C87-51 & HS \\
\hline
\end{tabular}

$\mathrm{RR}=$ reaction against $P$. melanocephala; $\mathrm{HS}=$ Highly susceptible; $\mathrm{S}=$ susceptible; INT = intermediate; $\mathrm{R}=$ resistant.

is due mainly to this hereditability, in the wider and narrower sense of the character. It must be pointed out that as results showed, it is possible to obtain resistant progeny from progenitors with susceptibility or intermediate behavior against $P$. melanocephala. This demonstrated the occurrence of transgressed segregations toward susceptibility and resistance as had been previously reported (Ramdoyal et al., 2000). The aforementioned study revealed that resistant progenitors produce the major proportion of resistant progeny and for this reason it is very important to incorporate resistant progenitors in breeding programs, but also intermediate or even susceptible progenitors can produce a certain proportion of resistant progenies. This reinforces the hypothesis that inheritance of resistance is of a polygenic nature (Costet et al., 2012) where, probably, effects of additive and genic interaction are manifested.

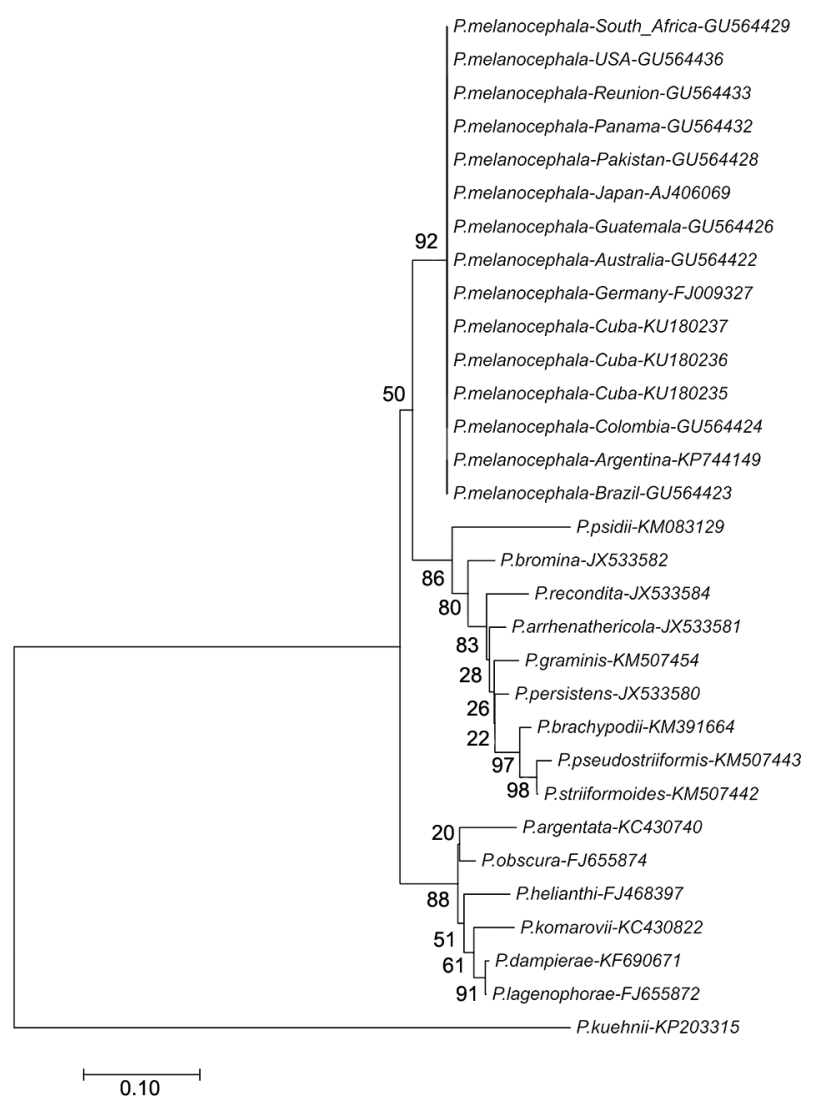

Figure $\mathbf{3}$ - Phylogenetic tree of Puccinia melanocephala from different countries and several species of the Puccinia genus based on partial ITS1, ITS2 and complete 5.8S nucleotide sequences generated with neighbor-joining option with a bootstrap analysis of 1,000 random replications.

In summary, the growing of varieties up to a limit of $20 \%$ of the sugarcane acreage in Cuba together with permanent phytosanitary monitoring of the cultivars and commercial release by the local sugarcane breed- 
ing program of genotypes of resistant or intermediate behavior against brown rust resulted in the avoidance of new epidemics. This reduced inoculum pressure even on susceptible and highly susceptible varieties that cannot occupy more than $10 \%$ of the planted area. This experience could be of great assistance to other sugarcane producer countries.

\section{Acknowledgements}

This project was supported by the Sugarcane Research Institute of Cuba (INICA), Agroindustrial Experimental Station "Obispo Colombres" (EEAOC - Argentina), National Council of Scientific and Technical Research (CONICET - Argentina) and National University of Tucuman (UNT - Argentina). M.F. Perera and A.P. Castagnaro are CONICET members and R.P. Bertani, a CONICET fellow.

\section{References}

Agrios, G.N. 2005. Plant Pathology. 5ed. Academic Press, Amsterdam, The Netherlands.

Aljanabi, S.M.; Forget, L.; Dookun, A. 1999. An improved and rapid protocol for the isolation of polysaccharide-and polyphenol-free sugarcane DNA. Plant Molecular Biology Reporter 17: 1-8.

Braithwaite, K.S.; Croft, B.J.; Magarey, R.C.; Scharaschkin, T. 2009. Phylogenetic placement of the sugarcane orange rust pathogen Puccinia kuehnii in a historical and regional context. Australasian Plant Pathology 38: 380-388.

Chu, T.L.; Serapion, J.L.; Rodríguez, J.L. 1982. Varietal reaction and inheritance trends of susceptibility of sugarcane to rust (Puccinia melanocephala Sydow \& P Sydow). Journal of Agriculture of the University of Puerto Rico 66: 99-108.

Costet, L.; Le Cunff, L.; Royaert, S.; Raboin, L.M.; Hervouet, C.; Toubi, L.; Telismart, H.; Garsmeur, O.; Rousselle, Y.; Pauquet, J.; Nibouche, S.; Glaszmann, J-C.; Hoarau, J-Y.; D'Hont, A. 2012. Haplotype structure around Bru1 reveals a narrow genetic basis for brown rust resistance in modern sugarcane cultivars. Theoretical and Applied Genetics 125: 825-836.
Glynn, N.C.; Dixon, L.J.; Castlebury, L.A.; Szabo, L.J.; Comstock, J.C. 2010. PCR assays for the sugarcane rust pathogens Puccinia kuehnii and P. melanocephala and detection of a SNP associated with geographical distribution in P. kuehnii. Plant Pathology 59: 703-711.

Hoy, J.W.; Hollier, C.A. 2009. Effect of brown rust on yield of sugarcane in Louisiana. Plant Disease 93: 1171-1174.

Koichiro, T.; Stecher, G.; Peterson, D.; Filipski, A.; Kumar, S. 2013. MEGA6: molecular evolutionary genetics analysis version 6.0. Molecular Biology and Evolution 30: 2725-2729.

Purdy, L.H.; Krupa, S.V.; Dean, J.L. 1985. Introduction of sugarcane rust into the Americas and its spread to Florida. Plant Disease 69: 689-693.

Raid, R.N.; Comstock, J.C. 2000. Common rust. p. 85-89. In: Rott, P.; Bailey, R.A.; Comstock, J.C.; Croft, B.J.; Saumtally, A.S., eds. A guide to sugarcane diseases. CIRAD/ISSCT, Montpellier, France.

Ramdoyal, K.; Sullivan, S.; Lim Shin Chong, L.C.Y.; Badaloo, G.H.; Saumtally, S.; Domaingue, R. 2000. The Genetics of rust resistance in sugar cane seedling populations. Theoretical and Applied Genetics 100: 557-563.

Rao, G.P.; Triphathi, D.N.P.; Upadhaya, U.C.; Singh, R.D.R.; Singh, R.R. 1996. CoSe-91423 and CoSe-93232: new promising red rot and smut resistant sugarcane varieties for eastern Utterpardesh. Indian Sugar Journal 46: 661-663.

Thompson, J.D.; Gibson, T.J.; Plewniak, F.; Jeanmouging, F.; Higgins, D.G. 1997. CLUSTAL X windows interface: Flexible strategies for multiple sequence alignment aided by quality analysis tools. Nucleic Acids Research 25: 4876-4882.

Townsend, G.R.; Heuberger, J.W. 1943. Methods for estimating losses caused by diseases in fungicides experiments. Plant Disease Reporter 27: 340-343. 